\title{
ROSAT PSPC view of the hot interstellar medium of the Magellanic Clouds ${ }^{\star}$
}

\author{
M. Sasaki, F. Haberl, and W. Pietsch
}

\author{
Max-Planck-Institut für extraterrestrische Physik, Giessenbachstraße, Postfach 1312, 85741 Garching, Germany
}

Received 15 March 2002 / Accepted 17 June 2002

\begin{abstract}
Diffuse X-ray emission from the Magellanic Clouds (MCs) is studied by using all the archival data of pointed ROSAT Position Sensitive Proportional Counter (PSPC) observations. For this purpose, contributions from the point and pointlike sources in the ROSAT High Resolution Imager (HRI) and PSPC source catalogues are eliminated and periods of high solar activity are excluded. The spectral analysis yielded characteristic temperatures of $10^{6}-10^{7} \mathrm{~K}$ for the hot thin plasma of the ISM which extends over the whole Large Magellanic Cloud (LMC) and the Small Magellanic Cloud (SMC). The total unabsorbed luminosity in the $0.1-2.4 \mathrm{keV}$ band within the observed area amounts to $3.2 \times 10^{38} \mathrm{erg} \mathrm{s}^{-1}$ in the LMC and $1.1 \times 10^{37} \mathrm{erg} \mathrm{s}^{-1}$ in the SMC, each with an uncertainty of $\sim-40 \%,+100 \%$. The X-ray luminosity of the LMC is comparable to that of other nearby galaxies with pronounced star formation. In the LMC, hot regions were found especially around the supergiant shell (SGS) LMC 4 and in the field covering SGS LMC 2 and LMC 3. Highest temperatures for the SMC were derived in the southwestern part of the galaxy. The diffuse X-ray emission is most likely a superposition of the emission from the hot gas in the interior of shells and supershells as well as from the halo of these galaxies.
\end{abstract}

Key words. shock waves - ISM: supernova remnants - galaxies: magellanic clouds - X-rays: ISM

\section{Introduction}

The small distances to the Magellanic Clouds (MCs) allow us to separate the emission from distinct sources from that arising from surrounding gas within the galaxies. Not only the discrete point sources and extended supernova remnants (SNRs), but also diffuse emission coming from the interstellar medium (ISM) can be observed and studied in detail. In the interstellar space, stars are born out of the densest regions, whereas massive stars transfer matter back to the ISM in stellar winds and supernova explosions. Therefore, the diffuse component of the $\mathrm{X}$-ray emission from galaxies will give us clues for a better understanding of the interaction between stars and the ISM as well as for the matter cycle within the galaxies.

With Einstein and ROSAT, high resolution X-ray imaging became possible and revealed diffuse X-ray emission in the Galaxy, the MCs, and other nearby galaxies (e.g. Fabbiano \& Trinchieri 1984; Watson et al. 1984; Snowden et al. 1991; Pietsch \& Kahabka 1993). It indicated the existence of a very hot component in the interstellar medium (ISM) with temperatures of $\sim 10^{6} \mathrm{~K}$ besides the cold gas observed in radio as well as warm components seen in the optical or UV. First supergiant shells (SGS) of relatively cold, ionized matter in the MCs

Send offprint requests to: M. Sasaki,

e-mail: manami@mpe.mpg. de

* Tables 2 and 3 are only available in electronic form at http://www. edpsciences.org were identified on $\mathrm{H} \alpha+\left[\mathrm{N}_{\text {II }}\right]$ images as filamentary structures (Goudis \& Meaburn 1978; Meaburn 1980). Shells are interpreted to result from matter swept up by expanding gas, and can be also seen in $\mathrm{H}_{\mathrm{I}}$ emission maps as dense regions around voids in the neutral hydrogen distribution.

Based on Einstein IPC data, very hot gas in the Large Magellanic Cloud (LMC) was mapped by Wang et al. (1991), and further detailed analysis of the diffuse X-ray emission was performed (e.g. Wang \& Helfand 1991a,b). SGS LMC 2 (Meaburn 1980) is one of the supergiant shells discovered in the LMC and is located next to the star formation region 30 Doradus. Wang \& Helfand (1991b) analyzed the Einstein IPC data of the SGS LMC 2 comparing it to infrared and $\mathrm{H}_{\mathrm{I}}$ observations, and found a ring of X-ray emission with a temperature of about $5 \times 10^{6} \mathrm{~K}$ within a cavity in the Hi map.

Bomans et al. (1994) analyzed ROSAT Position Sensitive Proportional Counter (PSPC) pointings covering the northern part of SGS LMC 4, which is located in the north of the LMC. They derived a temperature of $2.4 \times 10^{6} \mathrm{~K}$ by fitting a thermal plasma spectrum. As for SGS LMC 2, ROSAT and ASCA observations made it possible to measure a plasma temperature of $k T \approx 0.1-0.7 \mathrm{keV}$ (Points et al. 2000). Merged ROSAT PSPC images of the MCs were presented by Snowden \& Petre (1994) and Snowden (1999). For the LMC, 140 PSPC pointings were used and 20 for the Small Magellanic Cloud (SMC), and images were created in different spectral bands of the detector. In the LMC, a hot plasma was found along the optical bar with 
temperatures between $4 \times 10^{6}$ and $8 \times 10^{6} \mathrm{~K}$ increasing from west to east. In the SMC no pronounced diffuse X-ray emission was detected.

Both the LMC and the SMC were observed by ROSAT (Trümper 1982) in a period of over eight years in nearly 900 pointings, which covered the MCs almost completely. Therefore, a thorough study of the X-ray emission from the MCs has been started. The aim of this work was to establish a detailed picture of the high energy processes within a galaxy by producing a catalogue of sources in the MCs in the ROSAT band (see Haberl \& Pietsch 1999; Haberl et al. 2000; Sasaki et al. 2000a,b) and by analyzing the hot component of the interstellar medium. Since objects which can be detected in X-rays at the distances of the MCs are SNRs or binary systems including objects at the final stages of stellar evolution (supersoft sources, SSSs and X-ray binaries, XRBs), their distribution in combination with the structure and physical state of the ISM will indicate the region within the galaxies developing most actively and help us to understand the evolutionary history of the MCs. This work presents the spectral analysis of the complete ROSAT PSPC data unveiling the structure of the diffuse X-ray emission of the MCs with a spatial resolution of $15^{\prime} \times 15^{\prime}$.

\section{Data}

The archival data of the ROSAT PSPC are best suited for spectral analysis of the MCs as a whole, because there were pointed observations with an almost full coverage and high photon statistics. In total 223 pointings for the LMC in a field of $10^{\circ} \times$ $10^{\circ}$ around $\mathrm{RA}=05^{\mathrm{h}} 25^{\mathrm{m}} 00^{\mathrm{s}}, \mathrm{Dec}=-67^{\circ} 43^{\prime} 20^{\prime \prime} \quad(\mathrm{J} 2000.0)$ and 31 for the SMC in a field of $5^{\circ} \times 5^{\circ}$ around $\mathrm{RA}=01^{\mathrm{h}} 00^{\mathrm{m}} 00^{\mathrm{s}}$, Dec $=-73^{\circ} 00^{\prime} 00^{\prime \prime}(\mathrm{J} 2000.0)$ with single pointing exposure times of up to $65 \mathrm{ks}$ were analyzed using the EXSAS software package (Zimmermann et al. 1994).

Since the diffuse X-ray emission has a very low surface brightness and is extended over the field of view, it is important to eliminate the emission from point sources as well as the background emission very carefully. The background has to be modeled and can not be directly taken from the same observation as is common in point source analysis. In the course of spectral analysis, the background modeling is also one of the major topics, as will be shown in Sect. 3.2. But here, we first discuss the background components which can be eliminated during data preparation.

\subsection{Discrete sources}

Based on ROSAT PSPC and High Resolution Imager (HRI) catalogues of distinct X-ray sources in the MCs (Haberl \& Pietsch 1999; Haberl et al. 2000; Sasaki et al. 2000a,b), the contamination from all point and point-like X-ray sources in the ROSAT band was eliminated from the data. The extraction radii were computed according to the point spread function of the observations on the one hand, and the brightness and the extent of the sources on the other hand.

The ROSAT catalogues of discrete sources are complete to luminosities of $\sim 3 \times 10^{33} \mathrm{erg} \mathrm{s}^{-1}$. Very faint X-ray sources like cataclysmic variables or RS CVn stars with luminosities lower than $\sim 10^{33} \mathrm{erg} \mathrm{s}^{-1}$ are missing. In addition, there must be tens of old SNRs in the MCs which are still to be discovered, since the number of known SNRs in the MCs are smaller than what is expected (Wang et al. 1991). However, the number of discrete sources necessary to account for the diffuse X-ray emission in the LMC and the SMC would be $\sim 10^{5}$ and $\sim 10^{4}$, respectively (see Sect. 3.5.1), magnitudes higher than the expected number of missing sources. Thus the contribution of all the faint objects is small.

Furthermore, if the emission was a superposition of the emission from different sources, we would expect many thermal components which might result in a powerlaw spectrum. As will be shown in Sect. 3.3, the local spectrum of the diffuse $\mathrm{X}$-ray emission in each analyzed $0.25 \times 0^{\circ} .25$ region can be well reproduced by one thermal spectrum model. Therefore, the diffuse emission can mainly be attributed to hot interstellar gas.

\subsection{Background reduction}

In PSPC observations there is a contamination in lower pulse height channels due to afterpulse events (APE, Plucinsky et al. 1993; Snowden et al. 1994) which are thought to be caused by negative ion formation close to the anodes. We eliminated the APE by selecting out all events which occurred within $0.37 \mathrm{~ms}$ after and not further away than few arcmin from a preceding event in channels lower than 18.

Next, time intervals with higher background were screened out according to the housekeeping information of each observation. One part of the background is caused by X-rays of Solar origin scattering off the Earth atmosphere (Snowden $\&$ Freyberg 1993). The mainly contributing scattering mechanisms are Thomson scattering on oxygen, helium, and molecular nitrogen and fluorescent scattering of molecular nitrogen $(\mathrm{K} \alpha)$ and atomic oxygen $(\mathrm{K} \alpha)$. The oxygen column density $N_{\mathrm{O}}$ in the housekeeping data was used as an indicator for the Solar $\mathrm{X}$-ray background, giving the oxygen column density along the line of sight through the Earth's residual atmosphere. In order to estimate the values for the low, acceptable Solar X-ray background, time intervals with Solar zenith angle (angle between the Sun, the Earth, and the telescope) larger than $120^{\circ}$ were selected in the housekeeping data. This means that the satellite was in the shadow of the Earth and the effect of the Solar X-ray scattering was low. Thereafter the mean $\left\langle N_{\mathrm{O}}\right\rangle$ and the standard deviation $\sigma$ of $N_{\mathrm{O}}$ was computed for these nightside observations. With the help of these numbers, events in time intervals with $N_{\mathrm{O}}$ not exceeding the upper limit of $\left\langle N_{\mathrm{O}}\right\rangle+1 \sigma$ were finally selected from the event files.

Being high energy particle detectors, proportional counters are sensitive to cosmic rays as well. The particles falling through the detector also ionize the counter gas and cause signals. Therefore, the detector was equipped with an additional anode which was linked with the active detector anode in anticoincidence, and the vetoing during the observation was measured (master veto, Snowden et al. 1992). For preliminary particle background elimination, only intervals with master veto rate lower than $170 \mathrm{cts} \mathrm{s}^{-1}$ were chosen. Finally, we had to eliminate time intervals with enhanced count rates of unknown origin (short time and long time enhancements) which can be 
seen as an increase of the number of measured photons in the housekeeping data. For this purpose, mean values of the count rates $(60 \mathrm{~s}$ bin) were determined and intervals with count rate enhancements higher than $+1 \sigma$ were excluded.

\subsection{Creating spectra}

The cleaned event files of the single pointings were merged and binned for the SMC and the LMC resulting in a cheesed image for each of the MCs. In total, the LMC data contain 1990000 cts after the screening process, the SMC data 730000 cts. The mean exposure time for the LMC is $8 \mathrm{ks}$, the maximum exposure time $66 \mathrm{ks}$. In the SMC, the mean and maximum exposure times are $10 \mathrm{ks}$ and $43 \mathrm{ks}$, respectively. For a spatially resolved spectral analysis of the diffuse emission from the MCs, the data were divided into boxes of the size $0.25 \times 0.25$. Only boxes containing more than 1000 events were used for the following spectral analysis in order to have enough photon statistics. In Fig. 1 the merged data and the selected regions are shown.

In the reductional steps in Sect. 2.2, the particle background was eliminated only by excluding time intervals of high master veto rate. In order to completely dispose of the contribution of the high energy particles, their spectrum was modeled and subtracted from all the analyzed spectra. According to Snowden et al. (1992) and Plucinsky et al. (1993, 1996) the particle spectrum consists of three components. The first component is the internally produced spectrum of particles, measured when the filter wheel of the PSPC is closed by a $\sim 2 \mathrm{~mm}$ thick aluminum mask so that no X-rays are penetrating $\left(S_{\mathrm{FWC}}\right)$. There are two additional components which are measured when the filter wheel is open, but the detector out of focus: $\mathrm{Al} \mathrm{K} \alpha$ line at $\sim 1.5 \mathrm{keV}$ which is excited when the filter wheel is open and particles fall through the optical path of the telescope $\left(S_{\mathrm{Al}}\right)$, and the externally produced flat increase of the whole spectrum $\left(S_{\text {Ext }}\right)$. Since they are caused by infalling particles, they are all well correlated with the master veto rate. The components of the particle background spectrum as functions of the pulse height channel $\mathrm{CH}$ can be parameterized as follows:

$$
\begin{aligned}
S_{\mathrm{FWC}}= & {\left[39.66 \mathrm{CH}^{-2.91}-3.96 \times 10^{-6} \mathrm{CH}\right.} \\
& +0.0045] \times \mathrm{CH}^{-1} \\
S_{\mathrm{Al}}= & {\left[0.835 C H^{-0.75} \exp \left\{-0.716(12.247-\sqrt{C H})^{2}\right\}\right] \times C H^{-1} } \\
S_{\mathrm{Ext}}= & {\left[-4.47 \times 10^{-6} \mathrm{CH}+0.00493\right] \times C H^{-1} . }
\end{aligned}
$$

Particle background dominates in higher energy ranges, because the cosmic X-ray background is relatively low and the $\mathrm{X}$-ray mirror reflectivity of the telescope decreases significantly in these higher energy ranges. Therefore, counts in pulse height channels higher than 230 can be more or less ascribed to high energy particles and are used for normalizing the particle background spectrum. Finally we obtain for the total particle background:

$$
\begin{aligned}
S(M V, C H)= & {\left[\left(0.018+7.37 \times 10^{-4} M V\right) \times S_{\mathrm{FWC}}\right.} \\
& +\left(-0.004+2.29 \times 10^{-4} M V\right) \times S_{\mathrm{Al}} \\
& \left.+\left(0.007+2.21 \times 10^{-4} M V\right) \times S_{\mathrm{Ext}}\right] \times N_{230}
\end{aligned}
$$
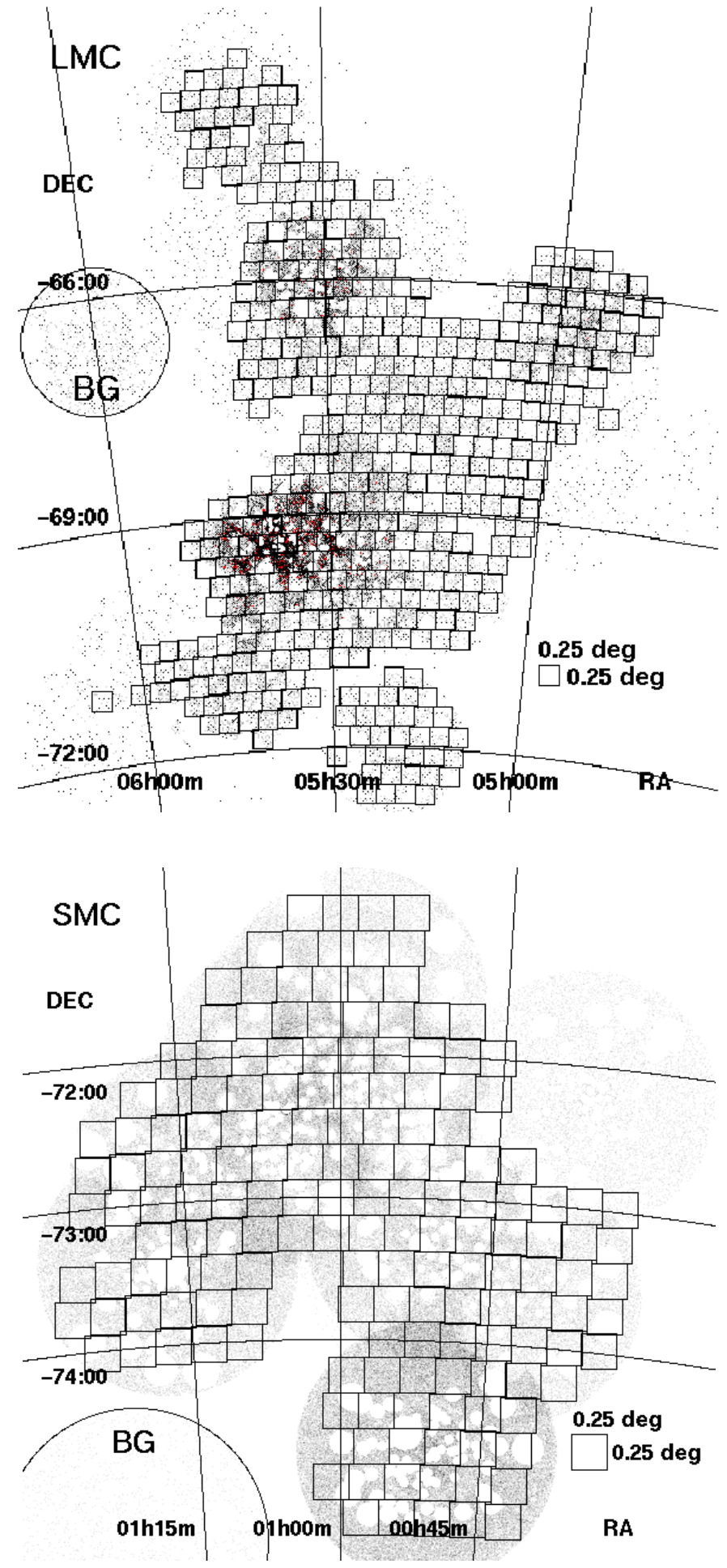

Fig. 1. Merged PSPC data of the MCs. Because the images are not exposure corrected, there are bright regions correlating with the pointings. Selected boxes are superimposed. Pointings used for the estimation of foreground and background emission are marked as "BG".

$M V$ : mean master veto rate;

$N_{230}$ : normalization of the spectrum with counts in channels higher than 230 . 
Events of the PSPC data were binned into spectra in each selected $0.25 \times 0.25$ box. The particle background spectrum was modeled for each spectrum taking the components $S_{\text {FWC,Al,Ext }}$ into account as defined in the Eqs. (1) to (3), and determining the master veto rate for the selected events. After normalizing with the number of events in the channels above 230, the modeled background spectrum (Eq. (4)) was subtracted from the total spectrum.

\section{Spectral analysis of the diffuse X-ray emission of the MCs}

\subsection{Absorption of X-rays by the ISM}

$\mathrm{X}$-rays traveling through the ISM are absorbed due to photoionization of atomic and molecular gas as well as interstellar dust grains. In the course of spectral analysis, the X-ray spectra obtained from observations must be corrected for these modifications. Latest studies of absorption of X-rays in the ISM are presented by Wilms et al. (2000). They discuss updated photoionization cross sections and abundances in the ISM and describe the effect of interstellar grains in detail.

For energies higher than $\sim 0.5 \mathrm{keV}$, the absorption is dominated by the heavier elements. Thus the X-ray opacity inferred from observations of $\mathrm{H}_{\mathrm{I}}$ is a lower limit, since substantial additional X-ray absorption is contributed by $\mathrm{He}, \mathrm{C}, \mathrm{N}, \mathrm{O}$, etc. in the ISM. Therefore the contributions of heavier elements must be considered, e.g. using the Solar metallicity for the Galactic ISM. The corrections for $\mathrm{H}_{2}$ clouds and grains along the line of sight are small and can be neglected unless spectral analysis of high accuracy (1-2\%) is carried out.

\subsection{Background and foreground components in the spectra}

Before modeling the spectral contribution of the MCs, components in the spectra coming either from the cosmic background or from the Galaxy in the foreground had to be determined. For this purpose, spectra of regions within the selected $10^{\circ} \times 10^{\circ}$ and $5^{\circ} \times 5^{\circ}$ fields including the LMC and the SMC, respectively, but distant enough from the MCs were analyzed first. For the LMC, pointings to the south ecliptic pole lying northeast of the LMC were suitable, for the SMC, pointings to the southeast of the SMC (see Fig. 1).

For the spectra, the cosmic background in the hard band and thermal emission from the Galactic foreground in the soft bands were taken into account. The cosmic background is well described by a highly absorbed power-law with photon index $\Gamma=1.4$ as e.g. ASCA observations have shown (Gendreau et al. 1995; Chen et al. 1997; Miyaji et al. 1998). It is absorbed by heavier elements both in the MCs (see below) and in the Galaxy which varies between $N_{\mathrm{HGal}}=3 \times 10^{20}$ and $8 \times 10^{20} \mathrm{~cm}^{-2}$ depending on position (Dickey \& Lockman 1990).

The foreground is described by two thermal components as a composition of the Local Bubble and the Galactic halo emission for which two Raymond \& Smith models (Raymond $\&$ Smith 1977) were used. Solar abundances were always assumed for Galactic components. The halo emission is absorbed

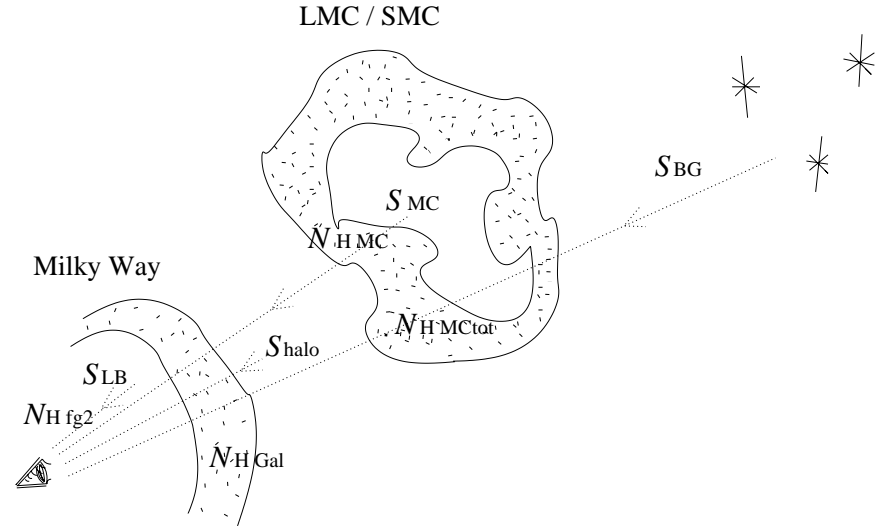

Fig. 2. Emission contributing to the analyzed spectrum. $S_{\mathrm{MC}}, S_{\mathrm{BG}}$, $S_{\text {halo, }}$, and $S_{\mathrm{LB}}$ are non-absorbed original emission components, $N_{\mathrm{H}}$ is the absorbing $\mathrm{H}$ column density.

by the Galactic $N_{\mathrm{Hfg} 1}=N_{\mathrm{HGal}}$, whereas the absorption of the Local Bubble emission $N_{\mathrm{H} \text { fg2 }}$ is about one order of magnitude lower. From ROSAT observations, the softest component was verified to be thermal with a plasma temperature between 0.05 and $0.09 \mathrm{keV}$ (Miyaji et al. 1998). In order to obtain the other parameters of the background+foreground spectrum, the spectra of regions as mentioned above were modeled using following components: 1) power-law for the cosmic background (fixed absorbing column densities $N_{\mathrm{HGal}}$ $\& N_{\text {HMCtot }}$ and fixed $\left.\Gamma\right), 2$ ) Raymond \& Smith spectrum for the Galactic halo (fixed absorption with $N_{\mathrm{Hfg} 1}=N_{\mathrm{HGal}}, k T_{\mathrm{fg} 1}$ to be determined), and 3) Raymond \& Smith spectrum for the Local Bubble $\left(0.05 \leq k T_{\text {fg2 }} \leq 0.09\right.$ and $N_{\mathrm{H} \text { fg2 }} \leq N_{\mathrm{HGal}}$ for the absorption). The fit of the spectra from regions close to the MCs resulted in $k T_{\mathrm{fg} 1}=0.18 \mathrm{keV}$ for the halo emission which is in good agreement with former ASCA and ROSAT results. For the Local Bubble component, the plasma temperature $k T_{\text {fg2 }}=0.09 \mathrm{keV}$ achieved the best fit. $N_{\mathrm{Hfg} 2}$ varies between 0.05 and $1.0 \times 10^{20} \mathrm{~cm}^{-2}$ for the LMC and between 0.25 and $0.90 \times 10^{20} \mathrm{~cm}^{-2}$ for the SMC. $N_{\mathrm{Hfg} 2}$ was set variable within these limits later on in spectral models including background, foreground, and MC emission. Furthermore, values for the emission measures $E M$ of the spectral components were obtained for the LMC and the SMC individually, and were later used for spectral fitting.

\subsection{Modeling the diffuse emission from the Magellanic Clouds}

All the spectra of the MCs were first fitted with a spectral model only containing contributions of the background and the foreground in order to verify whether there is additional emission or not. In the next step, the diffuse emission from the MCs was fitted with an additional absorbed Raymond \& Smith component yielding the characteristic temperature $T_{\mathrm{MC}}$ and the absorption $N_{\mathrm{HMC}}$. One of the most crucial tasks thereby was to find the best fitting interval for the temperature value. For $k T_{\mathrm{MC}}<0.1 \mathrm{keV}$, the foreground absorption of the X-rays becomes high, modifying the intrinsic luminosity by factors of about 10. Consequently, additional emission components with 


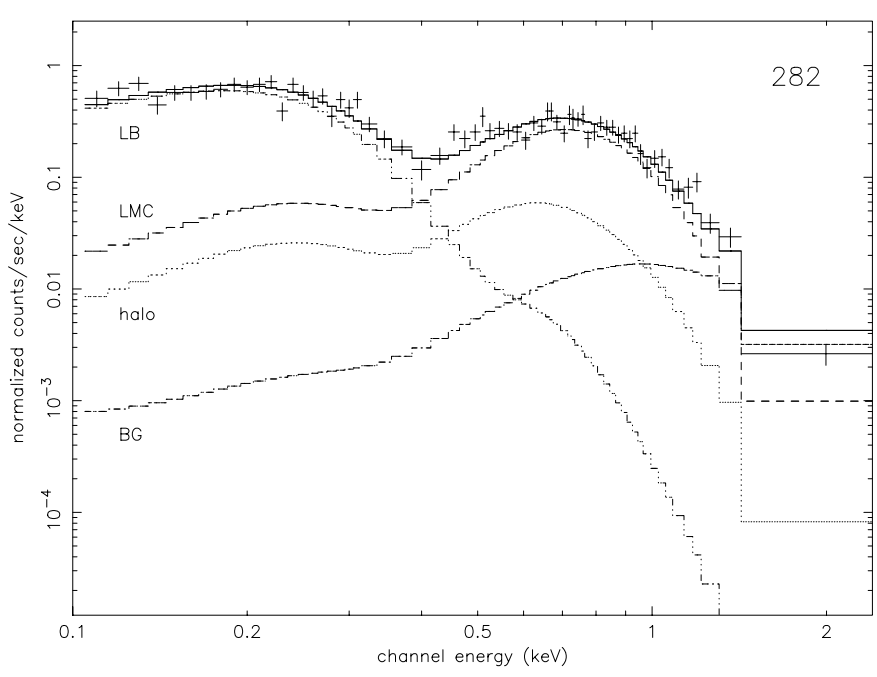

Fig. 3. Spectrum of the diffuse X-ray emission of the field No. 282 in the LMC. The model components for the extragalactic background, the Galactic halo, and the Local Bubble are plotted as well. The dashed line shows the LMC emission component.

small values of $T_{\mathrm{MC}}$ might be modeled, although in reality there is only very low additional emission which should be better ascribed to variations of the Galactic foreground emission. Therefore, the MC component was restricted to $k T_{\mathrm{MC}} \geq$ $0.1 \mathrm{keV}$.

As shown in Fig. 2, the total observed spectrum is

$S_{\mathrm{obs}}=S_{\mathrm{MC}}^{\mathrm{abs}}+\left(S_{\mathrm{BG}}^{\mathrm{abs}}+S_{\mathrm{halo}}^{\mathrm{abs}}+S_{\mathrm{LB}}^{\mathrm{abs}}\right)$

with

$$
\begin{aligned}
S_{\mathrm{MC}}^{\mathrm{abs}}= & \mathrm{e}^{-\sigma(E) N_{\mathrm{HGal}}^{\dagger}\left(\zeta_{\odot}^{\dagger}\right)} \times \mathrm{e}^{-\sigma(E) N_{\mathrm{HMC}}^{\diamond}\left(\zeta_{\mathrm{MC}}^{\dagger}\right)} \\
& \times S_{\mathrm{RS}}\left(T_{\mathrm{MC}}^{\diamond}, \zeta_{\mathrm{MC}}^{\dagger}, E M_{\square}^{\diamond}\right) .
\end{aligned}
$$

$S_{\mathrm{RS}}$ is the Raymond \& Smith model for thermal emission, for $S_{\mathrm{BG}}^{\mathrm{abs}}, S_{\text {halo }}^{\mathrm{abs}}, S_{\mathrm{LB}}^{\mathrm{abs}}$ see Eqs. (7), (8) and (9). In Eqs. (5) to (9) free parameters which were to be determined are marked with $\diamond$ and fixed parameters with $\dagger$. For the abundances in the MCs, correction factors $\zeta$ reported by Russell \& Dopita (1992) were used: 0.5 for the LMC, and 0.2 for the SMC (with respect to solar).

Again the Galactic foreground was considered as an additional component using the temperatures $T_{\mathrm{fg} 1}, T_{\mathrm{fg} 2}$, emission measures, and absorption column densities $N_{\mathrm{Hfg} 1}, N_{\mathrm{Hfg} 2}$ with Solar abundances as mentioned above:

$S_{\text {halo }}^{\text {abs }}=\mathrm{e}^{-\sigma(E) N_{\mathrm{HGal}}^{\dagger}\left(\zeta_{\odot}^{\dagger}\right)} \times S_{\mathrm{RS}}\left(T_{\mathrm{fg} 1}^{\dagger}, \zeta_{\odot}^{\dagger}, E M_{\mathrm{fg} 1}^{\dagger}\right)$,

$S_{\mathrm{LB}}^{\mathrm{abs}}=\mathrm{e}^{-\sigma(E) N_{\mathrm{Hfg} 2}^{\diamond}\left(\zeta^{\dagger}\right)} \times S_{\mathrm{RS}}\left(T_{\mathrm{fg} 2}^{\dagger}, \zeta_{\odot}^{\dagger}, E M_{\mathrm{fg} 2}^{\dagger}\right)$.

The cosmic background was modeled with the values for the photon index $\Gamma$ and the emission measure as determined previously, but the absorption by both the MCs $\left(N_{\mathrm{H} \text { MCtot }}\right)$ and the Galaxy $\left(N_{\mathrm{Hfg}}=N_{\mathrm{HGal}}\right)$ was taken into account:

$$
\begin{aligned}
S_{\mathrm{BG}}^{\mathrm{abs}}= & \mathrm{e}^{-\sigma(E) N_{\mathrm{HGal}}^{\dagger}\left(\zeta_{\odot}^{\dagger}\right)} \times e^{-\sigma(E) N_{\mathrm{HMCtot}}^{\dagger}\left(\zeta_{\mathrm{MC}}^{\dagger}\right)} \\
& \times S_{\text {pow }}\left(\Gamma^{\dagger}, n_{\text {pow }}^{\dagger}\right)
\end{aligned}
$$

$S_{\text {pow }}$ being the power-law model with normalization $n_{\text {pow }}$. For the total absorbing column density $N_{\mathrm{H} \text { MCtot }}$ of the MCs, values from the H i column density maps were used which were derived from the Australia Telescope Compact Array (ATCA) aperture synthesis mosaic survey and the $64 \mathrm{~m}$ Parkes single dish telescope observations (60" resolution for the LMC: Kim et al. 1998; Kim et al. 2001, and 98" for the SMC: Stanimirovic et al. 1999). In Fig. 3 a sample spectrum is shown with all the model components.

The existence of additional diffuse $\mathrm{X}$-ray emission from the MCs was verified for each $0.25 \times 0.25$ box, and the model yielding the lower reduced $\chi^{2}$ was chosen as the correct one. For all spectra with evidences for a MC component, the temperature $T_{\mathrm{MC}}$ of the emitting interstellar plasma, the local absorbing column density in the MCs $N_{\mathrm{HMC}}$, and the emission measure $E M_{\square}$ (see Eq.(11) for definition) for the analyzed boxes were obtained for both the LMC and SMC. The determined temperatures range from $0.1 \mathrm{keV}$ up to $1.5 \mathrm{keV}$ which means that in both MCs hot gas with temperatures from $10^{6} \mathrm{~K}$ to higher than $10^{7} \mathrm{~K}$ is found. The results of the modeling are listed in Tables 2 and 3 with box positions, absorption $N_{\mathrm{HMC}}$, temperature $T_{\mathrm{MC}}$, emission measure, and additionally derived physical parameters of the emitting gas.

Since each spectrum can be assigned to a certain region in the MC fields, images of the fit results were created by filling the square regions with derived temperature values $T_{\mathrm{MC}}$. They are shown in Fig. 4. In these images the pixel values were set to zero in regions without data. Therefore, the margin of the regions with $T_{\mathrm{MC}}>0$ is mainly caused by the fact that there was no or not enough data for the analysis in the adjacent regions (see Fig. 1). Also in Figs. 5 and 6, the regions with pixel values $>0$ are not characteristic for the extent of the total diffuse emission, but for the regions for which data was available.

\subsection{Errors of the spectral parameters}

For all spectral parameters of the diffuse X-ray emission from the MCs, uncertainties of the background and the foreground emission as well as the attenuation of X-rays by matter in the foreground cause a systematic error in addition to the statistical errors from the spectral fit. The statistical errors $\sigma_{\text {stat }}$ of the parameters $T_{\mathrm{MC}}, E M_{\square}$, and $N_{\mathrm{HMC}}$ were determined by fitting the spectra with all the other parameters fixed (background and foreground components, absorption of these components, and abundances). For $T_{\mathrm{MC}}$ the average statistical error $\sigma_{\text {stat }}$ is $-10 \%,+13 \%$, for $E M_{\square} \sim \pm 35 \%$, and for $N_{\mathrm{HMC}} \pm 57 \%$.

\subsubsection{Stray light}

In Fig. 4 one can see that in the very north of the LMC and in the very south, there are regions with high $T_{\mathrm{MC}}$. But as can be seen in Fig. 5 (see Sect. 3.5.1), the significance of the luminosity is relatively low. The high temperatures measured in these extended regions $\left(k T_{\mathrm{MC}} \geq 1.0 \mathrm{keV}\right)$ are caused by the highmass X-ray binary LMC X-3 in the north and the low-mass $\mathrm{X}$-ray binary LMC X-2 in the south. The stray light from these hard X-ray sources could not be eliminated from the analyzed 

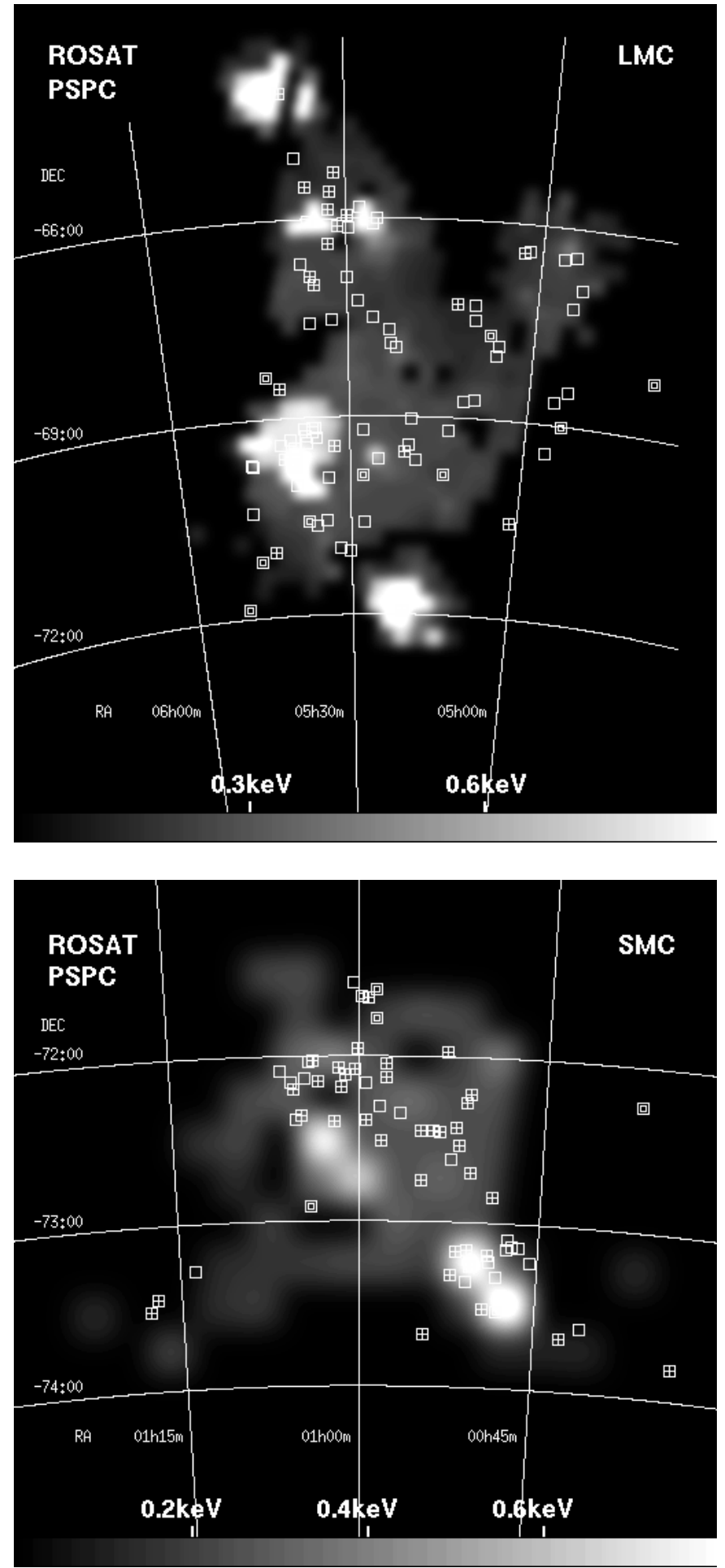

Fig. 4. Temperature distribution image of the LMC and the SMC. Square regions of the size $0.25 \times 0.25$ selected for spectrum extraction were filled with temperature values determined by spectral fitting. The image was then Gauss-filter smoothed. Positions of SNRs observed by ROSAT are shown by squares, those of XRBs by crossed squares, and for SSSs by double squares.

PSPC data, making both the temperature and the emission measure higher than what is typical for the hot interstellar gas.

\subsubsection{Systematic error}

The estimation of the background and foreground components in the spectrum was based on the work on cosmic X-ray background by Gendreau et al. (1995), Chen et al. (1997), and Miyaji et al. (1998). Since ASCA data was mainly used for the analysis presented by these authors, the spectral range which was studied extended up to $10 \mathrm{keV}$. Therefore, reliable information was given especially for the hard X-ray background: $\Gamma=1.4 \pm 0.1$. For the errors of the foreground components, the background+foreground emission spectra of regions mentioned in Sect. 3.2 were analyzed by varying the values for $\Gamma$ in the range specified by its error. In doing so, the errors of the temperatures of the foreground components were determined: $k T_{\text {fg1 }}=0.18_{-0.01}^{+0.05} \mathrm{keV}$ and $k T_{\text {fg2 }}=0.09 \pm 0.01 \mathrm{keV}$.

Background and foreground emission components are not the only sources of systematic error of spectral modeling, but the uncertainty of the strength of X-ray absorption by matter in the line of sight must be considered as well. As shown by Wilms et al. (2000), the correction for contributions by molecules and grains is negligible. However, if clouds are located in the line of sight, the absorption will be higher than estimated from the H i column density maps both for the Galaxy and for the MCs. Since the H I column density maps have moderate spatial resolution ( $1^{\circ}$ for the Galactic foreground), single clouds cannot be resolved, and the values used for the Galactic foreground absorption $\left(N_{\mathrm{Hfg}}\right)$ and the absorption of the cosmic background by the MCs $\left(N_{\mathrm{HMCtot}}\right)$ are average values.

In order to estimate the systematic error $\sigma_{\text {sys }}$ of the spectral analysis of the ISM of the MCs, all the uncertainties mentioned so far were taken into consideration and arbitrarily selected regions were fitted with all possible combinations of the lower

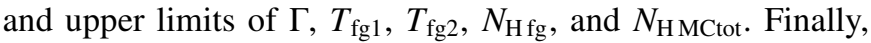
the systematic errors for the parameters of the spectral component assigned to the $\mathrm{MC}$ emission were obtained: for the temperature $T_{\mathrm{MC}}$ the systematic error $\sigma_{\text {sys }}$ is $\sim-5 \%,+8 \%$, for $E M_{\square} \sim-10 \%,+150 \%$, and for $N_{\mathrm{HMC}} \sim-5 \%,+20 \%$.

\subsection{Physical properties of the hot ISM}

\subsubsection{X-ray luminosity}

The unabsorbed luminosity of the gas in the $0.25 \times 0.25$ box regions of the MCs can be obtained from the spectral fit based on the model of Raymond \& Smith (1977). In the same way as the temperature images in Fig. 4 were created, images for the luminosity distribution were created by filling the square regions with $L_{\square}$ and smoothing them. The luminosity of the hot gas in a $0.25 \times 0.25$ region in the ROSAT band $(0.1-2.4 \mathrm{keV})$ ranges from $8.5 \times 10^{33}$ to $2.6 \times 10^{36} \mathrm{erg} \mathrm{s}^{-1}$ in the LMC and from $2.7 \times 10^{32}$ to $9.3 \times 10^{35} \mathrm{erg} \mathrm{s}^{-1}$ in the SMC. The total luminosity of the diffuse emission from the MCs was calculated by summing up the luminosities $L_{\square}$ of the $0.25 \times 0.25$ regions For the LMC, the obtained total luminosity is $3.2 \times 10^{38} \mathrm{erg} \mathrm{s}^{-1}$, for the SMC it is $1.1 \times 10^{37} \mathrm{erg} \mathrm{s}^{-1}$, both results with an uncertainty of $\sigma \approx-40 \%,+100 \%$. 

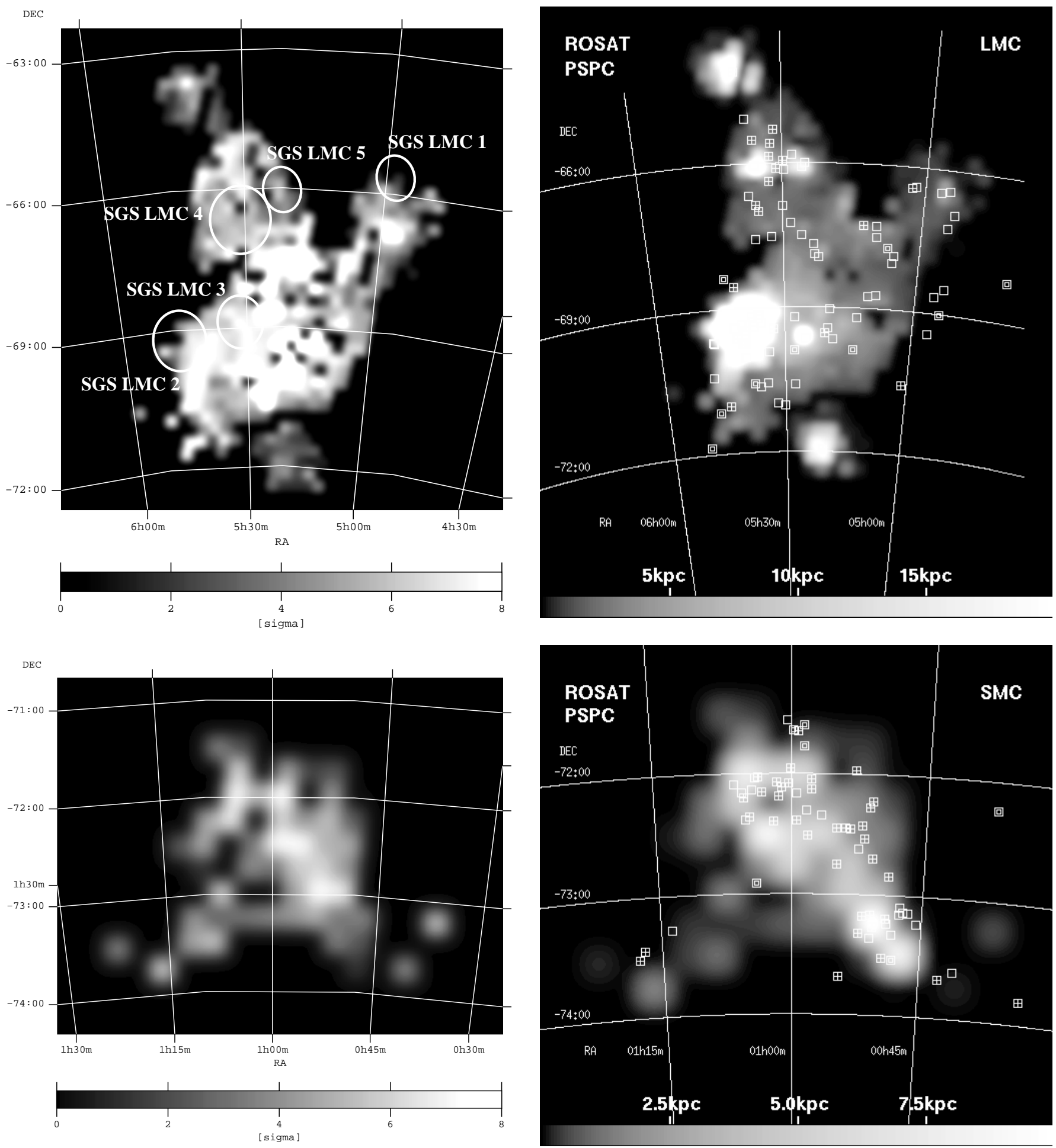

Fig. 5. Images showing the significance of the luminosity of the diffuse $\mathrm{X}$-ray emission. The luminosity was derived from the results of the spectral fit and divided by the $1 \sigma$ error. For the LMC the positions of the supergiant shells SGS LMC 1 to 5 are marked as ellipses.

Fig. 6. The distribution of the depth of the X-ray emitting regions in the MCs is shown. Similar to the temperature distribution images, after the regions used for spectrum extraction were filled with $\Delta l$, the images were smoothed. Symbols are the same as in Fig. 4.

\subsubsection{Geometry of the hot gas}

After the interstellar gas was heated up by a shock front, the

In Fig. 5 the significance of the luminosity determination of the diffuse emission is presented. The computed values of $L_{\square}$ are listed in Tables 2 and 3. high temperature is maintained because of the negligible cooling. Matter is mostly gathered to form a shell, so in its interior the gas can be treated as a perfect gas, and the density of the 
hot ISM can be estimated using the perfect gas equation. For coronal gas of cosmic abundances, the gas consists of ions and electrons, densities of which are related as $n_{\mathrm{e}}=1.213 n(\sim 10 \%$ He plus small fraction of heavier elements). Since the metalicity is lower than in the Solar neighborhood both for the LMC and SMC, it is $n_{\mathrm{e}}=(1.2+0.013 \zeta) n$. Therefore the number density for the gas is

$n=\frac{p}{(2.3+0.015 \zeta) k T}$

Assuming $p=3 \times 10^{-18}$ bar (typical value for the Galactic ISM, Spitzer 1990) and $T=10^{6} \mathrm{~K}$, the density is $n=0.01 \mathrm{~cm}^{-3}$.

The emission measure

$E M_{\square}=\frac{1}{10^{14} \times 4 \pi D^{2}} \int n_{\mathrm{e}} n \mathrm{~d} V\left[\mathrm{~cm}^{-5}\right]$,

for the $0.25 \times 0.25$ box was obtained from the spectral fit, with both the electron density $n_{\mathrm{e}}$ and gas density $n$ in $\left[\mathrm{cm}^{-3}\right]$ and $D$ being the distance to the source in $[\mathrm{cm}]$. We use $D_{\mathrm{LMC}}=$ $50 \mathrm{kpc}=1.54 \times 10^{23} \mathrm{~cm}$ and $D_{\mathrm{SMC}}=59 \mathrm{kpc}=1.82 \times 10^{23} \mathrm{~cm}$ (van den Bergh 1999). Since the emission measure depends on the radiating volume, it can be used to derive the depth of the $\mathrm{X}$-ray emitting region, while the pressure of the hot gas in the galaxy is assumed to be uniform. In fact, this is a crude assumption. If the whole galaxy was in pressure equilibrium, this would result in anticorrelation between the ISM temperature and the luminosity. As this is not observed, there must be pressure variations. However, assuming pressure equilibrium is a good starting point for the following estimation of the emitting gas volume, and will allow us to find deviating regions.

Spectra were extracted for regions of the size $0.25 \times 0.25$. Therefore, the emitting volume can be written as

$V_{\square}=A_{\square} \Delta l=\left(\frac{0.25}{360^{\circ}} 2 \pi D\right)^{2} \Delta l$.

$\Delta l$ is the thickness of the radiating layer. Based on Eqs. (11), (10), and (12) the depth $\Delta l$ is

$$
\begin{aligned}
\Delta l & =\frac{E M_{\square} \times 10^{14} \times 4 \pi D^{2}}{(1.2+0.013 \zeta) n^{2}}\left(\frac{720}{\pi D}\right)^{2} \\
& =\frac{4 \times(2.3+0.015 \zeta)^{2} \times 720^{2} \times 10^{14} \times k^{2}}{(1.2+0.013 \zeta) \pi p^{2}} E M_{\square} T_{\mathrm{MC}}^{2} \\
& =2.4 \times 10^{4} \times E M_{\square} T_{\mathrm{keV}}^{2}[\mathrm{kpc}]
\end{aligned}
$$

with $p=3 \times 10^{-18}$ bar and $E M_{\square}$ in $\left[\mathrm{cm}^{-5}\right] . T_{\mathrm{keV}}$ is the plasma temperature in $[\mathrm{keV}]$. A typical value of $E M_{\square}=0.001 \mathrm{~cm}^{-5}$ and $k T_{\mathrm{keV}}=0.3 \mathrm{keV}$ yield $\Delta l=2.2 \mathrm{kpc}$. Distributions of $\Delta l$ as derived from the fit values $T_{\mathrm{keV}}$ and $E M_{\square}$ for $p=3 \times 10^{-18} \mathrm{bar}$ are presented in Fig. 6 . The values for $\Delta l$ are also listed in Tables 2 and 3 .

In Fig. 6 one can see that $\Delta l$ is suspiciously high in the east of the LMC, including the $\mathrm{H}$ II region 30 Doradus as well as the SGSs LMC 2 and LMC 3 (see also Fig. 5). This is due to the wrong assumption that the pressure is the same as in the rest of the galaxy. In this very active region with a concentration of SNRs and stellar associations embedded in dense cold

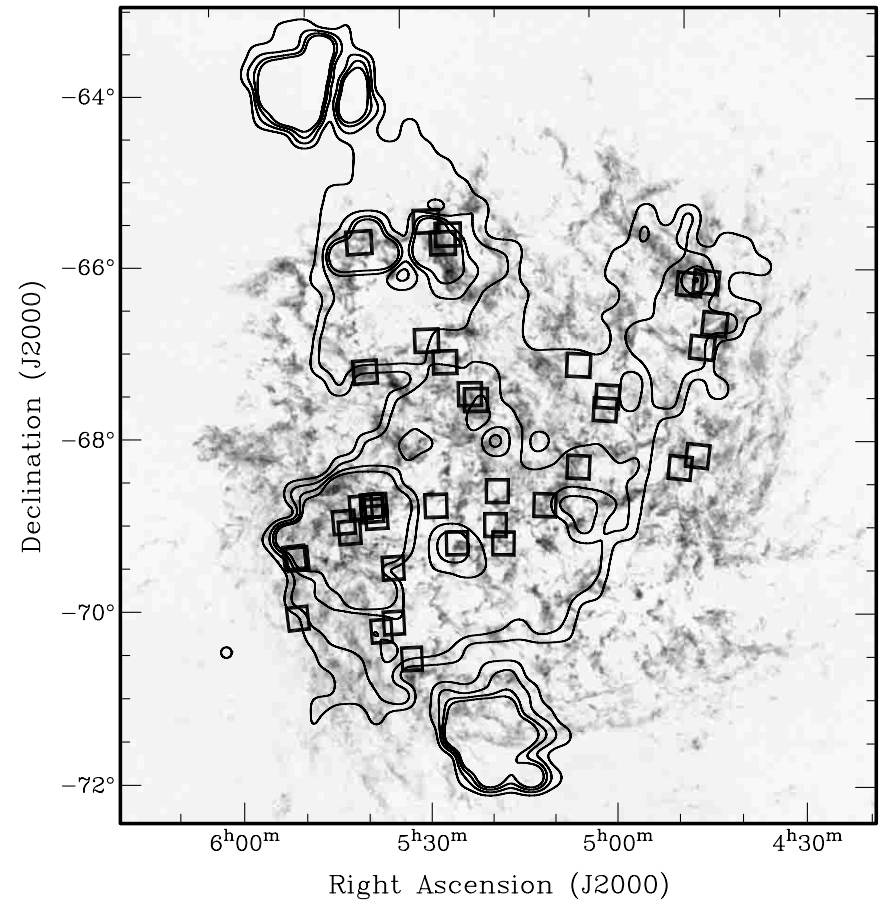

Fig. 7. Temperature contours from $0.1 \mathrm{keV}$ to $0.4 \mathrm{keV}$ in steps of $0.1 \mathrm{keV}$ are superimposed on a H I map of the LMC (Kim et al. 1998) with positions of SNRs observed by ROSAT marked as squares.

gas (see also Fig. 7), the pressure can be assumed to be higher, giving $\Delta l \propto p^{-2}$ a more realistic value.

The very high values for $\Delta l$ in the very north and in the very south of the LMC are consequences of the stray light of the bright sources LMC X-3 and LMC X-2 (see also Sect. 3.4.1).

\section{Discussion}

\subsection{Temperature distribution}

In order to verify the regions with significant diffuse X-ray emission and to look for correlations between the hot ionized gas and other components within the galaxies, the temperature distribution images (Fig. 4) were overlaid on other observations of the MCs, i.e. merged image from PSPC data, DSS image, or H i column density maps. Figure 7 shows the Hi column density map of the LMC with contours of X-ray temperatures, and in Fig. 8, the contours are plotted on a DSS image of the SMC.

\subsubsection{LMC}

In the LMC, SGSs were found in $\mathrm{H}$ I and $\mathrm{H} \alpha$ observations with diameters of the order of $1 \mathrm{kpc}$, i.e. each with a size of 5\%-10\% of the total size of the galaxy. The X-ray results show that there is an extended hot region in the eastern part of the LMC with a diameter of about $1 \mathrm{kpc}$, as can be seen in Fig. 4. This region covers the SGS LMC 2 (Meaburn 1980) where many active objects like SNRs and OB associations were found (see Figs. 4, 5, and 7). Using Einstein data, Wang \& Helfand (1991b) derived a luminosity of $\sim 2 \times 10^{37} \mathrm{erg} \mathrm{s}^{-1}$ for the diffuse emission from SGS LMC 2 assuming a temperature of $\sim 5 \times 10^{6} \mathrm{~K}$. Based on ROSAT PSPC data, Points et al. (2000) get a flux of 


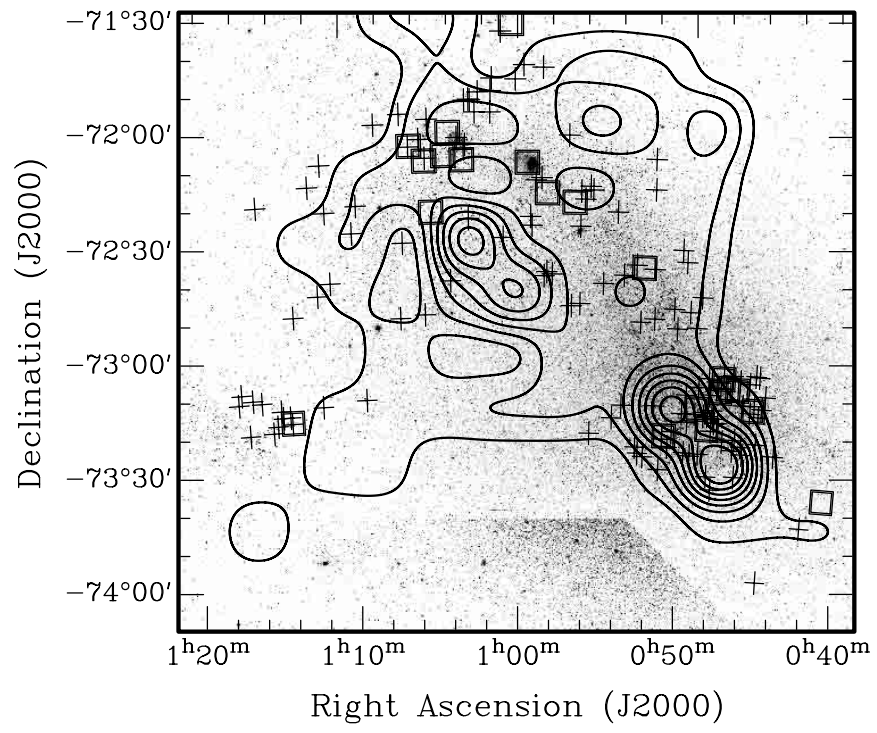

Fig. 8. Overlay of the temperature distribution as contours from 0.1 to $0.8 \mathrm{keV}$ in steps of $0.1 \mathrm{keV}$ on a DSS image of the SMC. Boxes are SNRs observed by ROSAT and crosses are $\mathrm{H}_{\text {II }}$ regions.

$1.4 \times 10^{-10} \mathrm{erg} \mathrm{cm}^{-2} \mathrm{~s}^{-1}$ in the energy band of 0.44 to $2.04 \mathrm{keV}$, which corresponds to $L_{\mathrm{X}}=6 \times 10^{37} \mathrm{erg} \mathrm{s}^{-1}(0.1-2.4 \mathrm{keV})$. They used a model with $k T=0.31 \mathrm{keV}$. In the same region, we find $L_{\mathrm{X}}=1.7 \times 10^{37} \mathrm{erg} \mathrm{s}^{-1}$ with a mean temperature of $k T_{\mathrm{MC}}=0.82 \mathrm{keV}$.

High temperature was also determined at the northern rim of SGS LMC 4 and the boundary between SGS LMC 4 and SGS LMC 5. At such boundaries between the hot ISM and the cold dense supergiant shells, the heating mechanism is most effective, since the expanding gas hits on dense matter and is strongly decelerated, the kinetic energy transforming itself to thermal energy. For SGS LMC 4, the integration of the X-ray luminosity gives $L_{\mathrm{X}}=1.6 \times 10^{37} \mathrm{erg} \mathrm{s}^{-1}$ and the mean value for the temperature is $k T_{\mathrm{MC}}=0.24 \mathrm{keV}$. This result is in a very good agreement with the work by Bomans et al. (1994), who obtained a temperature of $2.4 \times 10^{6} \mathrm{~K}$, i.e. $k T=0.21 \mathrm{keV}$ for the northern part of SGS LMC 4, as well as with the total luminosity of $\sim 2 \times 10^{37} \mathrm{erg} \mathrm{s}^{-1}$ for the SGS LMC 4 which was derived by Points et al. (2000) from the results of Bomans et al. (1994), assuming that the northern emission is representative for the whole SGS LMC 4.

As for the total diffuse emission of the LMC, Wang et al. (1991) determined a lower limit of $\sim 2 \times 10^{38} \mathrm{erg} \mathrm{s}^{-1}$ for the luminosity from Einstein data $(0.16-3.5 \mathrm{keV})$. The total luminosity derived from the ROSAT PSPC data in the energy band of 0.1 to $2.4 \mathrm{keV}$ is $3.2 \times 10^{38} \mathrm{erg} \mathrm{s}^{-1}$ (Sect. 3.5.1) with an error of $\sim-40 \%,+100 \%$, which is mainly due to uncertainties about the emission and absorption of the foreground gas.

\subsubsection{SMC}

The hot gas in the SMC is well correlated with the optical main body which can be seen on a DSS image with superimposed temperature contours (Fig. 8). Regions with the highest temperatures are found in the southwest part of the galaxy with a large number of SNRs and H II regions. The total X-ray emission not only arises from the optically visible part of the galaxy, but also shows the emission from the galactic halo. The existence of diffuse X-ray emission from the SMC was already reported by Wang (1991) based on the analysis of Einstein data who obtained a total X-ray luminosity of the diffuse emission $L_{\mathrm{X}}=5.0 \times 10^{38} \mathrm{erg} \mathrm{s}^{-1}$. But this was not verified in later ROSAT observations (Snowden 1999). As it was shown in Sect. 3.5.1, the ROSAT PSPC data yield a much lower luminosity $\left(L_{\mathrm{X}}=1.1 \times 10^{37} \mathrm{erg} \mathrm{s}^{-1}\right)$. A possible explanation for the discrepancy by a factor of about 50 between $L_{X}$ derived from Einstein observation and that of ROSAT is the incompleteness of the list of the point sources which were removed from the Einstein data, since a large number of new point sources were found in subsequent X-ray observations.

Radio observations revealed shells and giant shells within the SMC and in the outer regions (Staveley-Smith et al. 1997), but supergiant shells with large cavities like in the LMC are not known. The X-ray emission measured by the PSPC is the superposition of the emission from the interior of the shells which was heated up by stellar winds and supernovae.

\subsection{Comparison to stellar distribution}

In the LMC, stellar associations forming large scale systems were found (Shapley's constellations, McKibben Nail \& Shapley 1953) suggesting secondary star formation (Braunsfurth \& Feitzinger 1983; Isserstedt 1984). The most prominent system is the Shapley's constellation III, located in the north of the LMC. It coincides spatially with the SGS LMC 4 and includes a large number of OB stars in about 20 young associations. The formation of these stars is thought to be caused by the gravitational instability of the SGS LMC 4. Another well known star formation region in the LMC is the 30 Doradus region located at the border between SGS LMC 2 and LMC 3. The comparison of the distribution of young stars in the LMC $\left(t_{\text {age }} \leq 2 \times 10^{7} \mathrm{yr}\right.$, Isserstedt 1984) and the diffuse $\mathrm{X}$-ray emission shows that the hot gas is well correlated with the distribution of young stars. In regions with highest temperatures, i.e. around SGS LMC 4 or in the 30 Doradus region, there is a concentration of young supergiants $\left(t_{\mathrm{age}} \leq 8 \times 10^{6} \mathrm{yr}\right)$.

From optical observations it is known, that a young population of stars in the SMC is located along the bar (e.g. Brück 1980), and in particular concentrated in the southwest. In this part of the SMC, the hot gas detected in $\mathrm{X}$-rays coincides with the most active regions. This can be verified in Fig. 8 showing the positions of $\mathrm{H}_{\text {II }}$ regions, since associations of massive $\mathrm{OB}$ stars form $\mathrm{H}_{\text {II }}$ regions by their ionizing radiation and winds. Star forming regions are located in the eastern wing of the SMC as well, extending from the northern end to the east (Gardiner \& Hatzidimitriou 1992). Hot gas is distributed between the wing and the optical main body of the SMC, in a region around $\mathrm{RA}=01^{\mathrm{h}} 04^{\mathrm{m}}$, Dec $=-72^{\circ} 30^{\prime}$ surrounded by SNRs and $\mathrm{H}_{\text {II }}$ regions in the north. The wing region is thought to be caused by dynamical interactions between the SMC and the LMC and/or between the MCs and the Galaxy which triggered star formation 
(Murai \& Fujimoto 1980; Westerlund 1990). A concentration of gas in the wing region was probably formed by forces acting between the halos of the MCs, rather than by an outflow of interstellar material from the main body of the SMC.

\subsection{Diffuse emission from other nearby galaxies}

As was shown in the last sections, the diffuse X-ray emission of the MCs seems to arise from the halo on the one hand, and from regions with high star formation activity on the other hand. This is in good agreement with the models for stellar bubbles and the evolution of stars, predicting that the ISM is heated up by stellar winds and supernova explosions. The hot gas in the ISM can blow out of the galactic disk and flow into the halo. Diffuse X-ray emission as evidence for the ISM heating is also observed in other nearby galaxies. Some examples are listed in Table 1 with distance, $\mathrm{H}_{\mathrm{I}}$ mass, foreground $N_{\mathrm{H}}$, and X-ray luminosity.

NGC 1704 and NGC 1569 are Magellanic-type nearby galaxies with ongoing star formation activities. Their $\mathrm{H}$ I mass is comparable to that of the MCs (McGee \& Milton 1966; Stanimirovic et al. 2000). Similar to the MCs, there are bright star clusters embedded in superbubbles, and diffuse X-ray emission shows correlations with the $\mathrm{H} \alpha$ distribution. The unabsorbed X-ray luminosity of the diffuse emission is similar to that of the LMC (Hensler et al. 1998; Heckman et al. 1995). The face-on irregular galaxy NGC 4449 resembles the LMC as well, regarding its size, structure, and the diffuse X-ray emission (Vogler \& Pietsch 1997; Theis \& Kohle 2001). For the outer disk and the halo, a temperature of $\sim 3 \times 10^{6} \mathrm{~K}$ was measured for the interstellar plasma, created probably during the star formation between $2 \times 10^{6}$ and $4 \times 10^{7}$ years ago.

Similarity in diffuse X-ray luminosity is also observed between the LMC and spiral galaxies, like the edge-on spiral galaxy NGC 4631 which is known to have many $\mathrm{H}$ II regions and moderate star formation activity. The diffuse X-ray emission (Vogler \& Pietsch 1996) is about one order of magnitude higher than that of the LMC, as expected due to the bigger galaxy mass (Rand 1994). Like the MCs, the gas distribution in NGC 4631 is thought to have been influenced by tidal interactions with its neighboring galaxies (Combes 1978). In addition, supergiant H I shells like in the LMC were observed (Rand \& van der Hulst 1993).

One of the most famous nearby galaxies with X-ray emission studied in detail is the edge-on starburst galaxy NGC 253 with relatively low H i mass (Puche et al. 1991). Pietsch et al. (2000) analyzed the ROSAT data and found that the contributions of the nuclear area, disk, and halo to the diffuse X-ray emission are about equal. The nuclear area mainly consists of a heavily absorbed source with $k T=1.2 \mathrm{keV}$ and $L_{\mathrm{X}}=$ $3 \times 10^{38} \mathrm{erg} \mathrm{s}^{-1}$ and an "X-ray plume" described by two components $(k T=1.2 \mathrm{keV}$ and $k T=0.33 \mathrm{keV})$, which is thought to originate from the interaction between the galactic wind from the starburst nucleus and the interstellar medium within the disk. The halo emission is very soft with a temperature of $k T \approx 0.1 \mathrm{keV}$. In the spectra of the MCs, this component is difficult to be verified because of the higher Galactic foreground absorption.

Further spiral galaxies are known to show diffuse X-ray emissions, like the Seyfert 2 galaxy NGC 4258 (van Albada \& Shane 1975; Vogler \& Pietsch 1999) or the face-on spiral galaxy M 83 (Huchtmeier \& Bohnenstengel 1981; Ehle et al. 1998). These galaxies show at least two diffuse emission components, the soft halo emission and the hard, highly absorbed disk emission. Their diffuse $\mathrm{X}$-ray luminosity is very high $\left(L_{\mathrm{X}}>10^{40} \mathrm{erg} \mathrm{s}^{-1}\right)$ what is to be expected due to their star formation activity.

\section{Summary and outlook}

Analyzing the ROSAT PSPC data of pointed observations of the MCs, the temperature distribution of the hot component of the ISM in the MCs was determined with a $15^{\prime} \times 15^{\prime}$ resolution. The hot thin interstellar plasma in the LMC and the SMC has temperatures of $10^{6}$ to $10^{7} \mathrm{~K}$. In the LMC, highest temperatures were determined in regions including the SGSs LMC 2 and LMC 3, as well as in the northern part of SGS LMC 4. These are the regions with enhanced star formation rate where young massive stars were found. In the SMC, the interstellar gas is hottest in the southwestern part. Many SNRs were detected in this part of the galaxy, which is also known to have a large concentration of young stars. The results on the SMC is of particular importance, since its diffuse X-ray emission had not been studied in detail so far. Furthermore, the X-ray luminosity profile of the hot ISM was derived. The total luminosity within the observed area amounts to $3.2 \times 10^{38} \mathrm{erg} \mathrm{s}^{-1}$ in the LMC and $1.1 \times 10^{37} \mathrm{erg} \mathrm{s}^{-1}$ in the SMC with $\sigma \approx-40 \%,+100 \%$. The X-ray luminosity of the LMC is in the same order as that of other nearby galaxies which show diffuse emission. These galaxies have in common that their star formation rate is high. Based on the temperatures and emission measures as results of spectral modeling, the depth of the hot ISM was computed. The distribution of the hot ISM in the MCs shows that the highest temperatures are well correlated with secondary star formation, whereas the total hot gas is distributed over the whole galaxy.

The ISM is thought to be enriched and heated up during the stellar evolution, forming high-density shells of cold matter by compression. Nevertheless, the physical properties of the thin interstellar plasma are still not well understood. Thin highly ionized gas with magnetic fields comparable to those in the interstellar space is difficult to create and to be maintained in the laboratories on Earth. The new X-ray telescopes like XMM-Newton or Chandra with improved sensitivity and spectral resolution make it possible to study the thin plasma in interstellar space in full detail and determine its physical state. Furthermore, observations of other nearby galaxies in the near future will allow more elaborate studies of single objects and the ISM of galaxies. The comparison of the results from the analysis of other nearby galaxies to those of the MCs will give additional observational proofs for the matter recycling within the galaxies and will improve the understanding of the evolution of galaxies.

Acknowledgements. We would like to thank the anonymous referee for helpful comments. We are grateful to Drs. L. Staveley-Smith 
Table 1. Properties of diffuse emission from some other nearby galaxies.

\begin{tabular}{llccccl}
\hline \hline \multicolumn{1}{c}{ 1 } & \multicolumn{1}{c}{3} & 4 & 5 & 6 & \\
\hline \multicolumn{1}{c}{ Name } & Type & $\begin{array}{c}\text { Distance } \\
{[\mathrm{Mpc}]}\end{array}$ & $\begin{array}{c}\mathrm{H}_{\mathrm{I}} \text { mass } \\
{\left[10^{09} M_{\odot}\right]}\end{array}$ & $\begin{array}{c}N_{\mathrm{H}, \mathrm{Gal}} \\
{\left[10^{20} \mathrm{~cm}^{-2}\right]}\end{array}$ & $\begin{array}{c}L_{\mathrm{X}} \\
{\left[10^{39} \mathrm{erg} \mathrm{s}^{-1}\right]}\end{array}$ & \multicolumn{1}{c}{ References } \\
\hline SMC & SB(s)m & 0.06 & 0.4 & 5.1 & 0.01 & Stanimirovic et al. (2000), this work \\
NGC 1705 & SA0 & 5.0 & 0.09 & 3.5 & 0.12 & Hensler et al. (1998) \\
LMC & SB(s)m & 0.05 & 0.5 & 6.0 & 0.32 & McGee \& Milton (1966), this work \\
NGC 1569 & IBm & 2.2 & 0.2 & 22 & 0.4 & Heckman et al. (1995) \\
NGC 4449 & IBm & 3.7 & 1.0 & 1.2 & 1.0 & Theis \& Kohle (2001), Vogler \& Pietsch (1997) \\
NGC 4631 & SB(s)d & 7.5 & 7.0 & 1.2 & 4.0 & Rand (1994), Vogler \& Pietsch (1996) \\
NGC 253 & SAB(s)c & 2.6 & 1.0 & 1.3 & 4.0 & Puche et al. (1991), Pietsch et al. (2000) \\
NGC 4258 & SAB(s)bc & 6.4 & 5.0 & 1.2 & 20 & van Albada \& Shane (1975), Vogler \& Pietsch (1999) \\
M 83 & SAB(s)c & 8.9 & 20 & 4.0 & 36 & Huchtmeier \& Bohnenstengel (1981), Ehle et al. (1998) \\
\hline
\end{tabular}

Notes to

Col. 2: obtained from NASA/IPAC Extragalactic Database (NED).

Col. 5: galactic foreground $N_{\mathrm{H}}$ (Dickey \& Lockman 1990).

Col. 6: luminosity $(0.1-2.4 \mathrm{keV})$ of the diffuse emission from the galaxies corrected for Galactic foreground absorption.

and S. Kim for providing us with the $\mathrm{H}_{\mathrm{I}}$ column density maps of the LMC and the SMC. The ROSAT project is supported by the German Bundesministerium für Bildung und Forschung $(\mathrm{BMBF})$ and the Max-Planck Society. This research has made use of the NASA/IPAC Extragalactic Database (NED) which is operated by the Jet Propulsion Laboratory, California Institute of Technology, under contract with the National Aeronautics and Space Administration. We acknowledge the use of NASA's SkyView facility (http://skyview.gsfc.nasa.gov) located at NASA Goddard Space Flight Center. This research is based on photographic data obtained using The UK Schmidt Telescope. The UK Schmidt Telescope was operated by the Royal Observatory Edinburgh, with funding from the UK Science and Engineering Research Council, until 1988 June, and thereafter by the Anglo-Australian Observatory. Original plate material is copyright (c) the Royal Observatory Edinburgh and the Anglo-Australian Observatory. The plates were processed into the present compressed digital form with their permission. The Digitized Sky Survey was produced at the Space Telescope Science Institute under US Government grant NAG W-2166.

\section{References}

Allen, R. J., Goss, W. M., \& van Woerden, H. 1973, A\&A, 29, 447

Bomans, D. J., Dennerl, K., \& Kürster, M. 1994, A\&A, 283, L21

Braunsfurth, E., \& Feitzinger, J. V. 1983, A\&A, 127, 113

Brück, M. T. 1980, A\&A, 87, 92

Chen, L., Fabian, A. C., \& Gendreau, K. C. 1997, MNRAS, 285, 449

Combes, F. 1978, A\&A, 65, 47

Dickey, J. M., \& Lockman, F. J. 1990, ARA\&A, 28, 215

Ehle, M., Pietsch, W., Beck, R., \& Klein, U. 1998, A\&A, 329, 39

Fabbiano, G., \& Trinchieri, G. 1984, ApJ, 286, 491

Gardiner, L. T., \& Hatzidimitriou, D. 1992, MNRAS, 257, 195

Gendreau, K. C., Mushotzky, R., Fabian, A. C., et al. 1995, PASJ, 47, L5

Goudis, C., \& Meaburn, J. 1978, A\&A, 68, 189
Haberl, F., Filipović, M. D., Pietsch, W., \& Kahabka, P. 2000, A\&AS, 142,41

Haberl, F., \& Pietsch, W. 1999, A\&AS, 139, 277

Heckman, T. M., Dahlem, M., Lehnert, M. D., et al. 1995, ApJ, 448, 98

Hensler, G., Dickow, R., Junkes, N., \& Gallagher, J. S. 1998, ApJ, 502, L17

Huchtmeier, W. K., \& Bohnenstengel, H.-D. 1981, A\&A, 100, 72

Isserstedt, J. 1984, A\&A, 131, 347

Kim, S., Staveley-Smith, L., Dopita, M. A., et al. 1998, ApJ, 503, 674

Kim, S., Staveley-Smith, L., \& Sault, R. J. 2001, in Gas and Galaxy Evolution, ed. J. E. Hibbard, M. P. Rupen, \& J. H. van Gorkom (ASP Conf. Ser.)

McGee, R. X., \& Milton, J. A. 1966, Austr. J. Phys., 19, 343

McKibben Nail, V., \& Shapley, H. 1953, Proc. Nat. Acad. Sci., 39, 358

Meaburn, J. 1980, MNRAS, 192, 365

Miyaji, T., Ishisaki, Y., Ogasaka, Y., et al. 1998, A\&A, 334, L13

Murai, T., \& Fujimoto, M. 1980, PASJ, 32, 581

Pietsch, W., \& Kahabka, P. 1993, in New Aspects of Magellanic Cloud Research, 59-70

Pietsch, W., Vogler, A., Klein, U., \& Zinnecker, H. 2000, A\&A, 360, 24

Plucinsky, P. P., Snowden, S. L., Briel, U. G., Hasinger, G., \& Pfefferman, E. 1996, ApJ, 458, 861

Plucinsky, P. P., Snowden, S. L., Briel, U. G., Hasinger, G., \& Pfeffermann, E. 1993, ApJ, 418, 519

Points, S. D., Chu, Y.-H., Snowden, S. L., \& Staveley-Smith, L. 2000, ApJ, 545, 827

Puche, D., Carignan, C., \& van Gorkom, J. H. 1991, AJ, 101, 456

Rand, R. J. 1994, A\&A, 285, 833

Rand, R. J., \& van der Hulst, J. M. 1993, AJ, 105, 2098

Raymond, J. C., \& Smith, B. W. 1977, ApJS, 35, 419

Russell, S. C., \& Dopita, M. A. 1992, ApJ, 384, 508

Sasaki, M., Haberl, F., \& Pietsch, W. 2000a, A\&AS, 143, 391

Sasaki, M., Haberl, F., \& Pietsch, W. 2000b, A\&AS, 147, 75 
Snowden, S. L. 1999, in New Views of the Magellanic Clouds, IAU Symp., 190, 32

Snowden, S. L., \& Freyberg, M. J. 1993, ApJ, 404, 403

Snowden, S. L., McCammon, D., Burrows, D. N., \& Mendenhall, J. A. 1994, ApJ, 424, 714

Snowden, S. L., Mebold, U., Hirth, W., Herbstmeier, U., \& Schmitt, J. H. M. 1991, Science, 252, 1529

Snowden, S. L., \& Petre, R. 1994, ApJ, 436, L123

Snowden, S. L., Plucinsky, P. P., Briel, U., Hasinger, G., \& Pfeffermann, E. 1992, ApJ, 393, 819

Spitzer, L. J. 1990, ARA\&A, 28, 71

Stanimirovic, S., Staveley-Smith, L., Dickey, J. M., Sault, R. J., \& Snowden, S. L. 1999, MNRAS, 302, 417

Stanimirovic, S., Staveley-Smith, L., van der Hulst, J. M., et al. 2000, MNRAS, 315, 791

Staveley-Smith, L., Sault, R. J., Hatzidimitriou, D., Kesteven, M. J., \& McConnell, D. 1997, MNRAS, 289, 225

Theis, C., \& Kohle, S. 2001, A\&A, 370, 365
Trümper, J. 1982, Adv. Space Res., 2, 241

van Albada, G. D., \& Shane, W. W. 1975, A\&A, 42, 433

van den Bergh, S. 1999, in New Views of the Magellanic Clouds, IAU

$$
\text { Symp., 190, } 569
$$

Vogler, A., \& Pietsch, W. 1996, A\&A, 311, 35

Vogler, A., \& Pietsch, W. 1997, A\&A, 319, 459

Vogler, A., \& Pietsch, W. 1999, A\&A, 352, 64

Wang, Q. 1991, ApJ, 377, L85

Wang, Q., Hamilton, T., Helfand, D. J., \& Wu, X. 1991, ApJ, 374, 475

Wang, Q., \& Helfand, D. J. 1991a, ApJ, 373, 497

Wang, Q., \& Helfand, D. J. 1991b, ApJ, 379, 327

Watson, M. G., Stanger, V., \& Griffiths, R. E. 1984, ApJ, 286, 144

Westerlund, B. E. 1990, A\&AR, 2, 29

Wilms, J., Allen, A., \& McCray, R. 2000, ApJ, 542, 914

Zimmermann, H. U., Becker, W., Belloni, T., et al. 1994, EXSAS User's Guide (MPE report 257) 